\title{
Students' perceptions towards self-directed learning in Ethiopian medical schools with new innovative curriculum: a mixed- method study
}

\author{
Haftom Hadush Kidane ${ }^{1 *}$ (D) Herma Roebertsen ${ }^{2}$ and Cees P. M. van der Vleuten ${ }^{2}$
}

\begin{abstract}
Background: Self-directed learning (SDL) is an appropriate and preferred learning process to prepare students for lifelong learning in their professions and make them stay up-to-date. The purpose of this study was to explore preclinical students following a hybrid curriculum in Ethiopia experiences to SDL and the support of several learning activities from the curriculum on their SDL. A mixed-method research design was employed.

Methods: Quantitative data were collected by using a self-administered questionnaire of 80 items measuring students' perceptions on their SDL capability as well as to explore students' views about the influence of components of the curriculum on their SDL. Additional two focus group discussions, each containing eight participants from year-1 and year ${ }^{-2}$ students, were conducted. The quantitative data were analyzed using SPSS. The focus group discussions were reviewed, coded, and then thematically analyzed.

Results: Our study showed a significant increase in SDL score on comparing students at year- 1 with students at year-2 $(p=0.002)$. Both year-1 and 2 students rated PBL tutorial discussion and tutors had high influence on their individual learning; whereas, other curricular components such as lectures and testes had low influence on their SDL ability. PBL tutorial discussion and module objectives showed strong correlation with students' SDL scores, $r=0.718 \& r=0.648(p<0.01)$, respectively. Besides, PBL tutorial discussion was found strongly correlated with tutors $(r=0.599(p<0.01))$ and module objectives $(r=0.574(p<0.01))$. Assessment was highly correlated with lectures $(r=0.595(p<0.01))$. Findings from qualitative data showed that certain curricular components played role in promoting students' SDL. Tutorials analyzing problems played a major role on students' self-directed learning abilities.

Conclusions: Although the study implied that components of the hybrid curriculum, mainly PBL, could encourage preclinical students' self-directed learning, the curriculum is still not free from teacher-centred culture as the majority of teachers still have high power in deciding the learning process. A further longitudinal study is needed to verify the actual level and ability of medical students' SDL.
\end{abstract}

Keywords: Self-directed learning, Preclinical students, Hybrid curriculum, Ethiopia

\footnotetext{
* Correspondence: haftomki@yahoo.com

${ }^{1}$ Department of biomedical science, Aksum University, Aksum, Ethiopia

Full list of author information is available at the end of the article

(c) The Author(s). 2020 Open Access This article is distributed under the terms of the Creative Commons Attribution 4.0 International License (http://creativecommons.org/licenses/by/4.0/), which permits unrestricted use, distribution, and reproduction in any medium, provided you give appropriate credit to the original author(s) and the source, provide a link to the Creative Commons license, and indicate if changes were made. The Creative Commons Public Domain Dedication waiver (http://creativecommons.org/publicdomain/zero/1.0/) applies to the data made available in this article, unless otherwise stated.
} 


\section{Background}

Equipping medical professionals with skills essential for lifelong learning is immensely required [1]. In today's society, as scientific knowledge constantly develops, medical professionals should be able to learn and upgrade their competency from time to time [2]. Selfdirected learners have an adequate amount of accountability to select the content to be studied, to assign time for it, and to understand the content deeply using any method both inside and outside class [3]. Students mostly need training and support to become a selfdirected learner $[4,5]$. This support is necessary when students are teacher dependent and not ready at the beginning for self-directed learning (SDL) until they pass through a series of progressive stages [4].

To help learners persistently improve SDL skills, implementation of appropriate teaching strategies and activities has paramount importance [4]. Several studies have indicated different teaching strategies and activities that motivate students toward self-directed learning [6-8]. Problem-based learning (PBL) is one of such widely accepted educational strategies that stimulate SDL [8]. Evidence exists that PBL promotes SDL because learners are responsible to plan, monitor, and evaluate their learning process [9-11]. Within a PBL strategy, students usually work together in tutorials analyzing real-world problems [12]. These tutorial authentic problems promote students' independent responsibility for learning and help connect them with the large world beyond the classroom [12, 13].

To become a self-directed learner, there are promoting and deterring conditions related to the type of offered curriculum, and students' and teachers' level of understanding about SDL $[14,15]$. Unlike innovative curricula, a conventional curriculum is not likely to produce selfdirected learners [16]. A large body of research indicated graduates of $\mathrm{PBL}$ curricula are better self-directed learners $[17,18]$. On the other hand, there are inconsistent reports in different settings about the effect of a hybrid PBL curriculum on students' SDL ability [19]. By hybrid curriculum is meant a curriculum that combines traditional teaching methods and PBL aiming to benefit from advantages of the innovative learning strategy $[8$, 20]. Besides curriculum approach, there is no common consensus between the correlation of sociodemographic factors such as curriculum year, age, and academic achievement and students' SDL [21-28]. Leatemia et al. [25] revealed higher level of SDL readiness in first-year students compared to second-year medical students of a hybrid PBL curriculum. Novertheless, Frambach et al. [23] noted that students from three medical schools (two had PBL-curriculum and one had hybrid PBL curriculum) in different cultures progressively accustomed the principle of SDL from year to year. Premkumar and colleagues [28] indicated a significant effect of age on SDL ability: higher age medical students were more selfdirected learners compared with students from the same cohort of younger age. On the contrary, other studies have revealed no correlation between student age and SDL skill $[25,27]$. The relation between academic performance and SDL is still not clear; some studies reported strong correlation [22, 24, 26] while others reported weak relation [21].

The feasibility of SDL in all cultural learning environments has also been an area of discussion. Culture influences students' self-direction in learning [23, 29-33]. Thus, studying the effect of a hybrid curriculum on adult learners' SDL in the African setting is necessary.

So far, there are no studies conducted which investigate the effectiveness or level of self-directed learning in Ethiopian students in a hybrid curriculum like the New Innovative Medical Curriculum (NIMC) schools. The purpose of this study is, therefore, to explore how adult preclinical students' in Ethiopian culture experienced SDL and to see if they experienced the influence of the various elements of the hybrid curriculum on their SDL skill. We assumed that culture would influence SDL and year-two students to have higher levels of SDL ability than year-one students. Because year-one students are not ready for complete self-direction compared to students in year-two who are relatively with high experiences in PBL and supposed to pass through a series of progressive stages. Besides, we supposed that the effect of age and academic performance between students in year-one and year-two would be comparable. This study aims to provide information on the current lack of evidence on how students in Ethiopian medical schools with hybrid curriculum experience SDL. In addition, it contributes information for evidence-based decision of policymakers; it provides lesson for existing medical schools elsewhere to consider SDL; and it is a great input for future studies.

In the current study, several questions related to SDL are addressed: What do preclinical students who already have a bachelor degree understand by the term SDL? How do preclinical students from year-one reflect their level of SDL skill and does this differ from students in year-two? How do preclinical students from year-one perceive the support from the various elements of the hybrid curriculum on their SDL skills? Is this different from students in year-two?

\section{Methods}

\section{Setting}

The study was conducted at Aksum University medical school, Ethiopia. The medical school is among the 13 new medical schools in the country which adopted NIMC with the support of the Ethiopian Federal 
Ministries of Health and Education to tackle the significant shortage of doctors and burden of diseases in the country [34]. The medical curriculum is a five-year programme and designed for candidates aged below 35 years who already have a BSc degree in biomedical sciences. The first 2 years of the curriculum are preclinical years and the remaining 3 years are clerkship years [34]. It is a hybrid curriculum, some elements, such as weekly tutorial sessions are according to the PBL strategies, still, other elements, high number of lectures, stick more to traditional teacher-centred approach [34]. Case analyses in PBL tutorials are embedded in the first 2 years of the preclinical programme and students have to analyse multilevel PBL cases in a seven-step approach through tutorial one (pre-discussion), self-study, and tutorial two (reporting session) [12]. Multilevel PBL paper patient cases, which are constructed to address the core objectives of the preclinical years, are used to stimulate basic science learning. In the pre-discussion session $(2 \mathrm{~h}$ long) students follow the first five steps of the seven-step approach and end-up with designing learning goals. Between tutorials is time allotted for students' self-study to achieve all learning issues and get prepared for tutorialtwo discussion.

\section{Participants}

The study population was all year-one and year-two students of the medical school in Aksum. Almost all firstyear $(n=30$ from 31$)$ and second-year $(n=32$ from 38$)$ medical students of Aksum University participated in the study. In both years students follow comparable learning activities, a combination of PBL based learning activities, like each one tutorial with problem analyses and traditional based learning activities, like a weekly high number of teacher-centred lectures. In both year-1 and year-2, there are no training of other activities explicitly focusing on SDL. Data were collected after students completed all their course work, at the end of year-1 and year- 2 .

\section{Design}

We did a cross-sectional study [35] on first- and secondyear medical students at the School of Medicine. A mixed-methods research design [36] was employed to explore students' experience about their SDL skill and support of various elements of the curriculum on their SDL skill from March 26 - May 03/2019.

\section{Data collection}

The validated Likert-based self-rate scale for SDL (SRSSDL) questionnaire developed by Williamson [37] was used to measure students' perceptions of their SDL level. The questionnaire consists of 60 items related to students' level of self-directedness in learning and they are arranged in five domains: self-awareness, learning strategies, learning activities, self-evaluation, and interpersonal skills. The scale was found reliable with Cronbach's alpha coefficient for each domain varied between 0.71 and 0.79 [37]. Besides, the questionnaire designed by Dolmans \& Schmidt [14] was used to explore students' views about the influence of components of the hybrid curriculum on their SDL. The items are 20 in number and set in six themes: discussion in the tutorial group, content tested, course objectives, lectures, tutor, and references. The coefficient alpha for each theme was found between 0.51 and 0.82 [14]. All items were rated by students whether they behave always (5), often (4), sometimes (3), seldom (2), or never at all (1). A pilot study was conducted on four third-year medical students.

Two focus group discussions (FGDs), one containing eight participants from year-one and one containing year-two students were conducted using similar interview questions after the questionnaire survey was performed. The purpose of the FGDs was to get more insight and in-depth information about students' understanding of SDL and the influence of curricular components on their SDL. The focus group was designed following the guidelines for Stalmeijer \& colleagues [38] and its duration was ranged from 1:30-2:00 h long. Both FGD discussions were conducted in local language (Amharic) by two interviewers.

\section{Analysis}

Quantitative data were analyzed using IBM SPSS version 20 (IBM, Armonk, New York, USA). Mean and standard deviation of students' SDL scores were computed. Independent $t$-tests/ANOVA were used to investigate whether means of the scores differed between years, ages, marital status, and academic performance (cumulative grade point average, CGPA, of the particular year) of students. Students' level of self-directedness in learning was ranked as low SDL (if they score within 60-140 range), medium SDL (141-220 score), or high SDL (221-300 score) [37]. We analyzed all results using $\alpha=$ 0.05. Moreover, we used Pearson correlation to evaluate whether and how strongly curricular components and SDL skills were related.

The focus group discussions were audiotaped and transcribed. The discussion contents were translated to English language by one of the focus group interviewers and checked by another staff with good English language skill. Subsequently, it was reviewed, coded, and then thematically analyzed by the main author and the two interviewers of the focus groups. There were repeated discussions among coders until agreement was reached. 


\section{Ethical approval}

Ethical approval was obtained from the Institutional Review Board (IRB) of Aksum University College of Health Sciences. Also, study participants were asked to participate in the study voluntarily and written informed consent was obtained by all study participants.

\section{Results}

\section{Quantitative results}

\section{Overview of the personal aspects of participants}

The response rate was $97 \%(30 / 31 ; 26$ male, 4 female) for year-one and $84 \%(32 / 38 ; 28$ male, 4 female) for year-two students. The study population in year-1 and year-2 were comparable (Table 1$)$.

\section{Students' SDL ability}

The SDL readiness scale (SDLRS) questionnaire of year1 and year- 2 students was analyzed and compared. The overall mean SDL readiness score for preclinical students at Aksum University medical school was 225.63 $( \pm 34.11)$. Year- 1 students had a mean SDL score of 212.3 (medium level) whereas year-2 students had a mean score of 238.2 (high level) (Fig. 1). A significant increase in SDL score on comparing students at year-1

Table 1 Characteristics of year-1 \& year-2 respondents, Aksum University, School of medicine, 2019

\begin{tabular}{|c|c|c|}
\hline \multirow[t]{2}{*}{ Demographic factors } & Year-1 & Year-2 \\
\hline & $N=30$ & $N=32$ \\
\hline \multicolumn{3}{|l|}{ Age } \\
\hline $20-24$ & 2 & - \\
\hline $25-29$ & 28 & 27 \\
\hline $30-34$ & - & 5 \\
\hline \multicolumn{3}{|l|}{ Marital status } \\
\hline Single & 20 & 19 \\
\hline Married & 10 & 13 \\
\hline \multicolumn{3}{|c|}{ Recent background degree } \\
\hline BSC & 30 & 32 \\
\hline \multicolumn{3}{|l|}{ Background education } \\
\hline None health & 2 & 2 \\
\hline Medical Laboratory & 1 & 1 \\
\hline Midwifery & 6 & 4 \\
\hline Nursing & 5 & 13 \\
\hline Optometry & 1 & 1 \\
\hline Public Health officer & 15 & 10 \\
\hline Psychiatry & 0 & 1 \\
\hline \multicolumn{3}{|c|}{ Academic performance (CGPA) } \\
\hline$>3.5$ & 9 & 11 \\
\hline $3.0-3.5$ & 18 & 17 \\
\hline$<3.0$ & 3 & 4 \\
\hline
\end{tabular}

with students at year-2 $(p=0.002)$ was found in this study.

In both year-1 and year-2, there were no significant effects of age, marital status, and academic performance on students' SDL scores (Table 2).

\section{Influence of curricular components on students' SDL skills}

The perceived influence of tutorial discussion, content tested, module/case objectives, lectures, tutors, and dependence on suggested references/sources on the individual study were scored by both year-1 and year-2 students. Both year-1 and 2 students rated PBL tutorial discussion and tutors had a high influence on their independent learning; whereas, other curricular components such as lectures and testes had a low influence on their SDL ability (Table 3).

Correlation between curricular components and students' self-rated SDL scores were also executed. Tutorial discussion and module/case objectives showed a strong correlation with SDL scores. Regarding the relationship between curricular components, the tutorial discussion was correlated strongly with both tutors and module objectives. Content tested was highly correlated with lectures (Table 4).

\section{Qualitative results}

The focus group discussion from year- 1 and the focus group discussion from year-2 provided in-depth information about students' understanding of SDL and curricular components that support or hinder SDL. The findings are presented below, clustered in three themes: students' definition for SDL; support of the teacher of SDL; and curricular components and activities that influence students' SDL. Illustrative quotes are presented in italics.

\section{Students' definition for SDL}

Students were asked to share their definitions of SDL and it could be understood that both cohorts have a certain level of understanding about SDL. Compared to year-1, Year-2 students showed a deeper understanding of SDL. Year-2 students identified different aspects of SDL in their definitions. In their definition, the concept SDL was described as the ability to identify learning needs, to distinguish relevant references to meet the identified learning issues, to use appropriate learning strategies in achieving the identified learning gaps, and evaluating the effectiveness of the learning outcomes. The following quotation shows the general view on how year-1 students defined SDL:

'.... I think now SDL means to me digging out and expanding the small direction we got in the class.' [Year-1 student] 


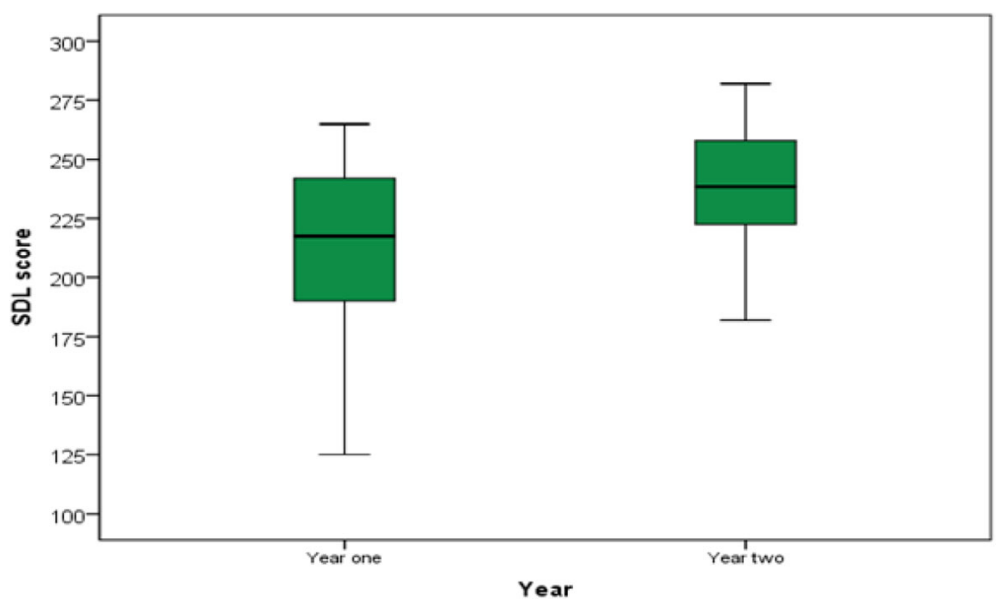

Fig. 1 Box plot demonstrating the mean SDL readiness score of year-1 \& year-2 students

\section{Support of the teacher of SDL}

The importance of support from teachers, particularly during year-one, is indicated in both focus groups as they played a vital role in making them self-directed learners.

'I think support from teachers is vital, especially at the beginning. We may be poor in identifying our gaps, may wish to read everything which is a problem for our survival, and may face difficulty how to get references..... For instance, I myself although completing year-1, I have still some problems how to use the internet and get reliable references.' [Year-1 student]

Table 2 A comparison of the SDL Scores of medical students within the year of study based on age, academic performance, \& marital status, Aksum University, School of medicine, 2019

\begin{tabular}{|c|c|c|c|c|}
\hline \multirow[t]{2}{*}{ Variables } & \multicolumn{2}{|l|}{ Year-1 } & \multicolumn{2}{|l|}{ Year-2 } \\
\hline & Mean score (SD) & $P$ value & Mean score (SD) & $P$ value \\
\hline Academic year & $212.3(36.26)$ & - & $238.2(26.90)$ & - \\
\hline \multicolumn{5}{|l|}{ Age } \\
\hline $20-24$ & $248.0(24.04)$ & 0.152 & - & 0.997 \\
\hline $25-29$ & $209.7(35.91)$ & & $238.2(28.18)$ & \\
\hline $30-34$ & - & & $238.2(21.23)$ & \\
\hline \multicolumn{5}{|c|}{ Academic performance } \\
\hline$>3.5$ & $213.3(37.87)$ & 0.934 & $252.5(19.86)$ & 0.066 \\
\hline $3.0-3.5$ & $213.0(36.59)$ & & $228.6(28.99)$ & \\
\hline$<3.0$ & $204.6(43.02)$ & & $239.2(20.17)$ & \\
\hline \multicolumn{5}{|l|}{ Marital status } \\
\hline Single & $213.1(39.96)$ & 0.862 & $236.3(28.53)$ & 0.648 \\
\hline Married & $210.6(29.34)$ & & $240.8(25.22)$ & \\
\hline
\end{tabular}

Interpretation of scores: Low (60-140); medium (141-220); high (221-300)

\section{Curricular components and activities that influence students' SDL}

Students in both cohorts mentioned several factors that facilitate or deter effective individual learning. Both year1 and 2 students indicated that, although they perceived they didn't have experience of SDL in their background education, the hybrid curriculum exposes them for individual learning and promotes their SDL ability as the tutorial session is incorporated within it. Students mentioned that their SDL is highly motivated by tutorial discussion and tutors because the discussion on cases helps them to identify learning gaps and as a consequence, they become inspired to read more on that. The participants expressed it as:

'As you guess, we didn't use SDL in our previous education. We used to expect everything from our teachers. SDL skill, as my colleagues said, needs background experience. I believe PBL tutorial discussion could break this barrier and made a great contribution to our current SDL ability. The tutorial discussion helped me to identify my learning needs and encouraged me to undergo individual study.' [Year-1 student]

I should say the guidance, support, and feedback provided by tutors inspired my SDL .... they frequently asked students to clarify things which were very helpful to recognize what we didn't clearly know.'[Year-2 student]

Both year-1 and year-2 students associate SDL largely with PBL which is illustrated by the following quotations of a year- 1 student followed by the quote of a year- 2 student. 
Table 3 Students' perceptions of the influence of the New Innovative medical Curriculum (NIMC) components on their SDL skills, Aksum University, School of medicine, 2019

\begin{tabular}{lclllll}
\hline $\begin{array}{l}\text { Class year of } \\
\text { students }\end{array}$ & \multicolumn{1}{l}{ Curriculum components } & & & & \\
\cline { 2 - 7 } & PBL tutorial discussion & Content tested & Module objectives & Lectures & Tutors & Reliance on suggested references \\
\hline Year-1 Mean (SD) & $3.5(1.13)$ & $1.95(0.52)$ & $2.9(1.03)$ & $1.9(0.6)$ & $3.7(1.04)$ & $1.78(0.6)$ \\
Year-2 Mean (SD) & $4.2(0.77)$ & $2.1(0.74)$ & $3.8(0.8)$ & $2.0(0.8)$ & $4.1(0.9)$ & $3.2(0.9)$ \\
\hline
\end{tabular}

$N B$ : Ratings were from 1 (minimum) to 5 (maximum)

'Actually, I started practising SDL since I joined this medical school which incorporates PBL. I didn't experience it in my background degree....' [Year-1 student]

'SDL is just having the motivation of teaching yourself on your own. It is exactly what we do in PBL..... you have learning issues which need further reading, so, you have to adjust your time and then select references to read ....... usually check myself whether I cover at least the most important learning issues because I want to actively participate in the tutorial discussion.'[Year-2 student]

Participants from year-1 and year-2 expressed the positive relation between SDL and PBL because, in the PBL session, they felt challenged and supported to feel responsible for own learning. In the same way, during PBL session they gradually built confidence to reflect their knowledge and understanding to colleagues in tutorials, classes, and elsewhere.

On the other hand, the influence of module objectives on students' SDL ability felt differently by year- 1 and year-2 cohorts. Students in year-2 perceived module objectives as a key element of the new innovative curriculum that supports individual learning because of its integrated nature in all modules. The explanations why this happened were indicated in the focus group discussions. Students from year- 1 and 2 stated their opinions:
'The module objectives have a positive influence on our SDL ability. I think this is due to the integration of basic sciences (multidisciplinary nature) in every module. This encouraged us to fully involved ourselves in connecting different points related to anatomy, physiology, microbiology, pathology, and pharmacology.' [Year-2 students]

'.....The introduction module which accounts for 16 weeks out of 45 weeks allocated for year-1 courses does not include PBL and mostly it is lecture-based which downgrade interactive sessions. I should say (because of this module) not that much' [Year-1 students]

Both year-1 and 2 cohorts similarly believed that curricular activities such as lectures seemed not motivating factors for self-directedness. It becomes clear that this curricular activity didn't foster students' SDL. Students perceived the curriculum is still dominated by lectures and it is not free from teacher-centred culture. Lectures were among the key factors that deterred SDL as indicated in the following statements:

'.... unfortunately, in my opinion, the main obstacle for our SDL was the lecture. Teachers tried to cover everything in non-interactive sessions and overwhelmed us with so many powerpoint slides, up to 2000, in a single course of a single system. Taking the tight schedule into account, reading all these notes

Table 4 Correlations among SDL scores and curricular components such as tutorial discussion, test, module objectives, lectures, tutors, and suggested references/sources, Aksum University, School of medicine, 2019

\begin{tabular}{lllllll}
\hline Variables & PBL tutorial discussion & Content tested & Module objectives & Lectures & Tutors & References/sources \\
\hline SDL skills & $.718 \mathrm{a}$ & .120 & $.648 \mathrm{a}$ & .085 & $.475 \mathrm{a}$ & $.420 \mathrm{a}$ \\
PBL tutorial- discussion & - & $.293^{\mathrm{b}}$ & $.574 \mathrm{a}$ & .147 & $.599 \mathrm{a}$ & $.366 \mathrm{a}$ \\
Content- tested & $.293^{\mathrm{b}}$ & - & .180 & $.595 \mathrm{a}$ & $.328 \mathrm{a}$ & .076 \\
Module- objectives & $.574 \mathrm{a}$ & .180 & - & .070 & $.412 \mathrm{a}$ & $.450 \mathrm{a}$ \\
Lectures & .147 & $.595 \mathrm{a}$ & .070 & - & .142 & .044 \\
Tutors & $.599 \mathrm{a}$ & $.328 \mathrm{a}$ & $.412 \mathrm{a}$ & .142 & - & .178 \\
References/sources & $.366 \mathrm{a}$ & .076 & $.450 \mathrm{a}$ & .044 & .178 & - \\
\hline
\end{tabular}

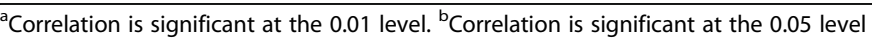


didn't give us time to go through reference books.' [Year-1 students]

'...........The lectures were poor in promoting us to identify our knowledge gaps.'[Year-2 students]

I think dumping a large number of lectures does not mean it is totally related to effective teaching-learning. Lectures have to be interactive, go with our level, cope-up with available time, and trigger SDL.'[Year-1 students]

Moreover, both year-1 and 2 focus groups felt that the assessment which was mainly lecture-based does not stimulate their SDL. Students believed lecture-based exams deterred their SDL.

'We were assessed mainly from lecture contents. To score high marks you had to be assessment-oriented and just reading your lecture notes and memorizing what you read.'[Year-2 students]

'To be honest, our exams were disrupting even for our PBL self-study.' [Year-1 students]

There was a similar perception of the impact of the country's higher education culture on SDL. Students indicated that their teachers are products of conventional curricula and the majority of them have high power in deciding what and how they learn.

'Teachers..... didn't see them to motivate us for individual learning. They rush to cover their bulky and tiresome lectures in the class. In this case..... I found teachers better when they facilitate tutorial than lecturing in the class.' [Year-1 students]

'Some of us are still with the mindset of high dependency on teachers since we thought teachers know everything about the course.' [Year-1 students]

I would say SDL had better be practised in other educational programs. Students joining the medical program could easily become popular with the method from the beginning.' [Year-1 students]

'Almost all of our teachers didn't have this (SDL) experience in their background education. I suggest all teachers should be involved in training like what has been done for tutors.' [Year-2 students]

\section{Discussion}

This study aims to explore self-directed learning (SDL) ability of preclinical students and to assess how various elements of a hybrid curriculum influence students' SDL. Year-1 and year-2 students are compared.

The overall students' average SDL score was 225.63 which indicate high self-direction. In our study, firstand second-year students self-rated their SDL skill, 212.3 (medium level) and 238.2 (higher level), respectively. Significant differences were observed in SDL scores on comparing students at year-1 with students at year-2. This implied students' SDL ability differed with a year of study. Our findings are in line with the study that revealed students from PBL curriculum to be progressively better self-directed learners across curricular years [17]. Nevertheless, our findings differ from a previous crosssectional study on a hybrid curriculum at Dalhousie University Faculty of Medicine, Canada that shows no differences in students' SDL scores between year-1 and 2 [19]. Our results are also different from cross-sectional studies on hybrid curricula in University of Toronto Faculty of Medicine and Indonesian medical schools where first-year medical students had significantly higher scores than second-year students [25, 39]. Besides, other longitudinal studies that followed undergraduate students' SDL readiness in PBL hybrid and traditional medical curricula showed a significant drop in SDL readiness scores with medical training $[28,40]$. The observed SDL differences between years in the present study might be due to the progressive positive effect of the NIMC, particularly PBL tutorials, on students' SDL skills. Findings from focus group discussions show that SDL, particularly SDL in PBL, supports students to feel responsible for their learning. Lack of prior SDL experience in students' background education might be a reason for first-year students to perceive a relatively lower level of SDL than those in year-2. Compared to year-1, students from year-2 provided the main elements of the SDL concept in their definitions showing some differences in practising SDL.

The findings of this study showed no significant effect of age on students' SDL scores. This is similar to different studies elsewhere [25, 27]. On the contrary, it disagrees with other studies reported older students had significantly higher scores than younger students [28, 41]. Our finding probably implies students enrolled in the NIMC program have comparable age as all are within the adult population with a background degree. In adult learning theory older students are supposed to be with an equivalent ability of planning, conducting, and evaluating own learning [42].

It was noticed that there is no significant effect of academic performance (GPA) on students' SDL scores. It is supported with the previous studies [21, 28, 43, 44] 
and disagreed with several studies reporting that SDL was found to be related to students' academic performance $[22,24,26]$. A possible explanation for our finding might be the lecture-oriented test practised with great emphasis on fact learning and no room to assess the level of insight and reasoning as discussed within the tutorials in the medical school couldn't promote students' self-directed learning. According to Shah et al. [45], medical students may sometimes show a tendency to exercise a surface learning approach.

Regarding the influence of curricular elements on students' SDL scores, PBL tutorial discussion and tutors were self-rated by students for their high influence on independent learning. Because of this, students generally perceived the hybrid curriculum promoting their SDL. The finding is in line with the report of Lee et al. [19] that stated a hybrid curriculum positively influence students' SDL skills. However, our result is different from those of Harvey et al. [39] who reported the effect of a hybrid curriculum at the Faculty of Medicine, University of Toronto, Canada, didn't foster students' SDL. From the findings of qualitative data, both year-1 and 2 students indicated, although they perceived they didn't experience SDL in their background education, the New Innovative Medical Curriculum exposed them for autonomous learning and promotes their SDL ability as the preclinical curriculum contains PBL within it. Students' SDL was highly motivated by tutorial discussion and tutors. The discussion on cases provides students with many clues and helped them to identify learning gaps for independent learning [46]. Our study shows other curricular elements such as lectures and testes that had low influence on students' SDL ability. This was supported with findings from focus group discussions which revealed these curricular activities that were not perceived by students as motivating factors for self-directedness. Students felt that the teaching-learning culture was still dominated by lectures and lectureoriented tests. The teaching-learning system was not free from teacher-centred culture since the majority of teachers still had high power in deciding the learning process. Our findings disagreed with the study in Canada by Lee et al. [19] who reported students' SDL ability was highly influenced by lectures. This difference might be due to the variation in the learning environment and lecture skills of faculty in both settings. A large body of literature suggests that teachers have to practice facilitation role more than the transmission of information $[47,48]$. This reminds us of the importance of having teachers equipped with skills essential to implement SDL promoting curriculum [49].

Regarding the correlation among the curricular components and students' SDL scores, tutorial discussion and module objectives showed a strong correlation with
SDL scores. These quantitative findings suggested that both discussions in the tutorial group and module objectives powerfully related to students' self-directedness. This finding would appear to differ with a study reporting that tutorial discussion and unit/case objectives showed a weak correlation with students SDL ability [19]. In the focus group interview, the influence of module objectives on students' SDL ability felt differently by year-1 and year- 2 cohorts. Students in year-2 identified module objectives as a key element of the curriculum that supports individual learning because of its multidisciplinary and integrated nature in all year- 2 modules. Students in year- 1 explained all modules of year- 1 didn't promote their SDL: for example, the 'Introduction to Medicine' module was perceived as less integrated and interactive. Furthermore, the tutorial discussion was strongly correlated with both tutors and module objectives. Our study noted that content tested was highly correlated with lectures. This finding implies that students tend to rely more on the content covered in lectures and tests [14].

This study has several limitations. It is limited because of a small sample size and lack of participants from teachers, although almost all preclinical students were encouraged to participate in our study. Its focus on a single Ethiopian medical school may limit the generalizability of the findings across different medical schools in the country and beyond. Another most important limitation is linked to the study design employed. The current study describes the students' SDL ability at one time without following up their progression. Following students throughout the academic year or across years could better ascertain students' progress in having SDL than a cross-sectional study. Moreover, because SDL ability was assessed by students themselves, it might limit the reliability of the findings. To address comprehensively, future research should consider the limitations observed in the current study to deeply investigate students' SDL ability and benefits of hybrid curricular innovations.

\section{Conclusion}

In the present study, first- and second-year medical students self-rated their SDL score as medium and higher level, respectively and significant differences are observed in comparing across years. The findings of this study show no significant effect of age and academic performance on students' SDL scores. Some components of the hybrid curriculum are perceived as promoting SDL. PBL tutorial discussion and tutors show high influence on students' independent learning; whereas, other curricular components such as lectures and tests show a low influence on students' SDL. Tutorial discussion and module objectives show a strong correlation with 
student's SDL ability. Tutorial discussion is correlated strongly with both tutors and module objectives. Content tested is highly correlated with lectures and this implies students tend to rely more on the content covered in lectures and tests which does not influence on SDL. Findings from qualitative data demonstrate that certain curricular components played a role in promoting students' SDL. Tutorials analyzing problems played a major role in students' self-directed learning abilities. Taken as a whole, the present study shows the New Innovative Medical Curriculum (NIMC) is still not free from teacher-centred culture since the majority of teachers still have high power in deciding the learning process and since all modules are still not well integrated and interactive. As most of the Ethiopian teachers are products of conventional curricula, continuous training may play a supportive role in making them skillful how to implement SDL promoting curriculum and how to promote students SDL. Most importantly, a further longitudinal study is needed to independently verify the actual level and behaviours of medical students' SDL in a hybrid curriculum.

\section{Abbreviations \\ CGPA: Cumulative grade point average; NIMC: New innovative medical curriculum; PBL: Problem-based learning; SDL: Self-directed learning; SDLRS: Self-directed learning readiness scale; SRSSDL: Self-rate scale for self- directed learning}

\section{Acknowledgements \\ I would like to thank Dr. Emmaline Brouwer who was the manager of the NUFFIC project (ETH-218) that granted my Master of Health Professionals Education (MHPE) at Maastricht University. I thank all students who participated in this study. I would also like to thank Hailay Gebreyesus and Getachew Mebrahtu for their support in leading the focus group discussions.}

\section{Authors' contributions}

$\mathrm{HH}$ involved in designing the study, collecting the data, analyzing the data, carrying out data analysis, interpretation of findings and writing the manuscript. HR and CPMvdV contributed to the study methodology, supervised interpretation of findings and corrected the final version of the manuscript. All authors read and approved the final manuscript.

\section{Authors' information \\ Haftom Hadush, MSc, Master in Health Professionals Education (MHPE), is an assistant professor and department head of the Medical laboratory science at Aksum University college of health science, Ethiopia [email: haftomki@yahoo.com]. \\ Herma Roebertsen is a Senior Lecturer and Educational adviser at Maastricht University, Faculty of Health, Medicine, and Life Sciences, Maastricht University, The Netherlands [email: h.roebertsen@maastrichtuniversity.nl]. Ceesd P.M. van der Vleuten, PhD, is a professor of Medical Education, Department of educational development and research at Maastricht University, Faculty of Health, Medicine, and Life Sciences, Maastricht University, The Netherlands [email: c.vandervleuten@maastrichtuniversity.nl].}

\section{Funding}

The authors declare that they have no source of funding for the research.

\section{Availability of data and materials}

The datasets used and/or analysed during the current study are available from the corresponding author on reasonable request.

\section{Ethics approval and consent to participate}

This study received ethical approval from the Institutional Review Board (IRB) of Aksum University College of Health Sciences (Number: IRB 127/2019). All participants were asked to participate in the study voluntarily and gave written informed consent before participation.

\section{Consent for publication}

Not applicable.

\section{Competing interests}

The authors declare that they have no competing interests.

\section{Author details}

'Department of biomedical science, Aksum University, Aksum, Ethiopia. ${ }^{2}$ Department of Educational Development and Research, Faculty of Health, Medicine and Life Sciences, Maastricht University, Maastricht, The Netherlands.

Received: 17 September 2019 Accepted: 30 December 2019

Published online: 08 January 2020

\section{References}

1. Murad MH, et al. The effectiveness of self-directed learning in health professions education: a systematic review. Med Educ. 2010;44(11):1057-68.

2. Laal M, Salamati P. Lifelong learning; why do we need it? Procedia - Soc Behav Sci. 2012:31:399-403.

3. Kaufman $D M$. ABC of learning and teaching in medicine: applying educational theory in practice. BMJ. 2003;326(7382):213-6.

4. Grow GO. Teaching learners to be self-directed. Adult Educ Q. 1991; 41(3):125-49.

5. Loyens SMM, Magda J, Rikers RMJP. Self-directed learning in problem-based learning and its relationships with self-regulated learning. Educ Psychol Rev. 2008:20(4):411-27.

6. Gade S, Chari S. Case-based learning in endocrine physiology: an approach toward self-directed learning and the development of soft skills in medical students. Adv Physiol Educ. 2013;37(4):356-60.

7. Janotha B. The effect of team-based learning on student self-directed learning. Ann Arbor: ProQuest LLC; 2015.

8. Savin-Baden M, Major CH. Foundations of problem-based learning. Maidenhead: Society for Research into Higher Education \& Open University Press; 2004.

9. Dolmans DHJM, et al. Problem-based learning: future challenges for educational practice and research. Med Educ. 2005;39(7):732-41.

10. Norman GR, Schmidt HG. The psychological basis of problem-based learning: a review of the evidence. Acad Med. 1992;67(9):557-65.

11. Silén C, Uhlin L. Self-directed learning - a learning issue for students and faculty! Teach High Educ. 2008;13(4):461-75.

12. Wood DF. ABC of learning and teaching in medicine: problem based learning. BMJ. 2003;326(7384):328-30.

13. Delisle R. How to use problem-based learning in the classroom. Alexandria: ASCD; 1997.

14. Dolmans $\mathrm{DH}$, Schmidt $\mathrm{H}$. What drives the student in problem-based learning? Med Educ. 1994;28(5):372-80.

15. Lunyk-Child Ol, et al. Self-directed learning: faculty and student perceptions. J Nurs Educ. 2001;40(3):116-23.

16. Spencer JA, Jordan RK. Learner centred approaches in medical education. BMJ. 1999:318:1280. https://doi.org/10.1136/bmj.318.7193.1280.

17. Schmidt, H.G., L. Vermeulen, and H.T.v.d. Molen. Longterm effects of problem-based learning: A comparison of competencies acquired by graduates of a problem-based and a conventional medical school. Medical Education vol. 40 no. 6, pp. 562-567, 2006. DOl: https://doi.org/10.1111/j. 1365-2929.2006.02483.x.

18. Shin JH, Haynes RB, Johnston ME. Effect of problem-based, self-directed undergraduate education on life-long learning. CMAJ. 1993;148(6):969

19. Lee YM, Mann KV, Frank BW. What drives students' self-directed learning in a hybrid PBL curriculum. Adv Health Sci Educ. 2010;15(3):425-37.

20. Armstrong EG. A hybrid model of problem-based learning. Challenge Probl Based Learn. 1997:2:137-50.

21. Abraham RR, et al. Exploring first-year undergraduate medical students' self-directed learning readiness to physiology. Adv Physiol Educ. 2011; 35(4):393-5. 
22. Bhat PP, Rajashekar B, Kamath U. Perspectives on Self-Directed Learning - the Importance of Attitudes and Skills. Bioscience Education. 2007;10(1):1-3.

23. Frambach $\mathrm{JM}$, et al. Rethinking the globalisation of problem-based learning: how culture challenges self-directed learning. Med Educ. 2012;46(8):738-47.

24. Khiat $\mathrm{H}$. Academic performance and the practice of self-directed learning: the adult student perspective. J Furth High Educ. 2017;41(1):44-59.

25. Leatemia LD, Susilo AP, van Berkel $H$. Self-directed learning readiness of Asian students: students perspective on a hybrid problem based learning curriculum. Int J Med Educ. 2016;7:385-92.

26. Lounsbury JW, et al. An investigation of the construct validity of the personality trait of self-directed learning. Learn Individ Differ. 2009;19(4):411-8.

27. Phillips BN, Turnbull BJ, He FX. Assessing readiness for self-directed learning within a non-traditional nursing cohort. Nurse Educ Today. 2015;35(3):e1-7.

28. Premkumar $\mathrm{K}$, et al. Does medical training promote or deter self-directed learning? A Longitudinal Mixed-Methods Stud Acad Med. 2013;88(11):1754-64.

29. Ahmad BE, Majid FA. Self-directed learning and culture: a study on Malay adult learners. Procedia - Soc Behav Sci. 2010;7:254-63.

30. Chan S. The Chinese Learner--A Question of Style. Education + Training. 1999;41(6-7):294-304.

31. Guglielmino PJ, Guglielmino LM. Culture, Self-Directed Learning Readiness, and Per Capita Income in Five Countries. SAM Adv Manag J. 2006;71(2): 07497075 .

32. Manikutty S, Anuradha N, Hansen K. Does culture influence learning styles in higher education? Int J Learn Chang. 2007;2(1):70-87.

33. Merriam SB. Andragogy and self-directed learning: pillars of adult learning theory. New Dir Adult Contin Educ. 2001;2001(89):3-14.

34. Abraham Y, Azaje A. The new innovative medical education system in Ethiopia: background and development. Ethiop J Health Dev. 2013;27(1):36-40.

35. Kumar R. Research methodology : a step-by-step guide for beginners. 3rd ed. London: Sage; 2011.

36. Creswell JW. Research design: qualitative, quantitative, and mixed methods approaches. Thousand Oaks: Sage publications; 2014

37. Williamson SN. Development of a self-rating scale of self-directed learning. Nurse Res. 2007;14(2):66-83.

38. Stalmeijer RE, McNaughton N, Van Mook WN. Using focus groups in medical education research: AMEE guide no. 91. Med Teach. 2014;36(11):923-39.

39. Harvey BJ, Rothman Al, Frecker RC. Effect of an undergraduate medical curriculum on students' self-directed learning. Acad Med. 2003;78(12):1259-65.

40. Premkumar K, et al. Self-directed learning readiness of Indian medical students: a mixed method study. BMC Med Educ. 2018;18(1):134.

41. Knowles MS, editor. The modern practice of adult education : from pedagogy to andragogy. Rev. and updated. Englewood Cliffs: Cambridge Adult Education; 1980.

42. Taylor DC, Hamdy H. Adult learning theories: implications for learning and teaching in medical education: AMEE guide no. 83. Med Teach. 2013;35(11):1561-72.

43. Balamurugan $\mathrm{S}$, Kumar $\mathrm{H}$. Self-directed learning readiness (SDLR) among medical students: a questionnaire-based study from an Indian medical school. South East Asian J Med Educ. 2015:9(2):59-64.

44. Deyo ZM, et al. Readiness for self-directed learning and academic performance in an abilities laboratory course. Am J Pharm Educ. 2011;75(2):25.

45. Shah DK, et al. Learning approach among health sciences students in a medical college in Nepal: a cross-sectional study. Adv Med Educ Pract. 2016;7:137.

46. Hmelo-Silver CE. Problem-based learning: what and how do students learn? Educ Psychol Rev. 2004;16(3):235-66.

47. Harden RM, Crosby J. AMEE guide no. 20: the good teacher is more than a lecturer--the twelve roles of the teacher. Med Teach. 2000;22(4):334-47.

48. Iwasiw CL. The role of the teacher in self-directed learning. Nurse Educ Today. 1987;7(5):222-7.

49. Knowles MS. Self-directed learning : a guide for learners and teachers. New York: Cambridge, the Adult Education Co; 1975.

\section{Publisher's Note}

Springer Nature remains neutral with regard to jurisdictional claims in published maps and institutional affiliations.

\section{Ready to submit your research? Choose BMC and benefit from}

- fast, convenient online submission

- thorough peer review by experienced researchers in your field

- rapid publication on acceptance

- support for research data, including large and complex data types

- gold Open Access which fosters wider collaboration and increased citations

- maximum visibility for your research: over $100 \mathrm{M}$ website views per year

At BMC, research is always in progress.

Learn more biomedcentral.com/submissions 